\title{
The Energetics of Horizontal Convection
}

\author{
R. W. Griffiths and G. O. Hughes \\ Research School of Earth Sciences \\ The Australian National University, Canberra ACT 0200, AUSTRALIA
}

\begin{abstract}
Convection driven by a gradient of temperature or heat flux along one horizontal boundary produces a localized turbulent plume and an otherwise stably stratified circulation. Informed by laboratory observations of the steady-state convection, we examine the rate of production of available potential energy, rate of viscous dissipation and rate of conversion of turbulent kinetic energy to potential energy by vertical mixing. Given the heat flux through the flow, the requirement of a steady state in the interior determines the vertical turbulent diffusivity in the interior, and an expression is also found for the boundary layer thickness. Applied to the zonally-averaged meridional overturning of the oceans, where we allow for a turbulent transport coefficient at the sea surface, the energy arguments lead to a thermocline depth of $300-530 \mathrm{~m}$. For the estimated meridional heat flux of $2 \times 10^{15} \mathrm{~W}$, we predict an average diffusivity of $\overline{\kappa^{*}}=\mathrm{O}\left(10^{-5}\right) \mathrm{m}^{2} / \mathrm{s}$, in agreement with measurements of vertical mixing. Also in agreement with previous estimates and ocean observations is the predicted rate of production of available potential energy $\left(0.5 \times 10^{12} \mathrm{~W}\right)$, of which $0.8-1.5 \times 10^{11}$ $\mathrm{W}$ is used to maintain the density structure.
\end{abstract}

\section{Introduction}

Horizontal convection driven by a horizontal gradient of temperature or heat flux along the top or bottom boundary of a volume of fluid is remarkably different from the (more extensively studied) convection forced by a temperature difference applied between either two vertical boundaries or the bottom and top boundaries. In horizontal convection, the heat flux enters a horizontal boundary layer and is extracted at the same boundary, with little heat passing through the bulk of the interior of the volume. The heat flux is, however, achieved through a horizontal flow in the boundary layer. There must be a return flow, and in experiments using a monotonic temperature gradient along the base or surface of a rectangular box, this is observed to occur as a vertical buoyant plume at one end of the box, nearest the destabilizing heat flux $[21,22,13]$. The plume extends through the full depth of the box (although this aspect is sensitive to nonsteady boundary conditions and side wall heat loss [13]). More gentle vertical flow, perhaps distributed through the volume, returns the fluid to the boundary layer in the region of stabilizing heat flux. The boundary layer dynamics have been described by Rossby [21]. However, it is not clear how the boundary fluxes drive a deep vigorous overturning, and how the interior maintains a steady state given an interior density stratification (or a buoyancy flux in the plume through all levels, as is required for the plume to penetrate through the depth).The global thermohaline circulation (or meridional overturning circulation) of the oceans is one possible, and particularly important, example of horizontal convection. Surface waters of the oceans are subject to net heating at equatorial latitudes (between approximately $20^{\circ} \mathrm{S}$ and $23^{\circ} \mathrm{N}$ based on a global annualized average) and to net cooling polewards of these latitudes [4]. The circulation of surface waters carries a net poleward transport of heat to maintain the balance, and highly localized downwelling at polar latitudes carries surface waters to abyssal depths $[24,21,14,19]$.
Much of the heat transport is a consequence of wind-driven surface flows. However, it is not clear whether the heat transport is passive $[15,5,27,28]$, or whether instead the thermal buoyancy flux associated with the meridional heat flux is a significant contribution to the driving forces in the momentum budget for the mean overturning flow. A separate question is whether the internal vertical mixing (which maintains the density structure of the oceans in the presence of the vertical components of the circulation) is substantially sustained by energy input from buoyancydriven flow (hence by the heat flux) or whether the mixing is dominated by energy from tides and winds [14, 15, 5, 27, 28].

In current diffusive theories of the meridional overturning circulation the density structure throughout much of the ocean is maintained by a balance between slow upwelling of cold water and downward turbulent mixing of heat, for which the required vertical diffusivity (at least at depths between $1000 \mathrm{~m}$ and $3000 \mathrm{~m})$ is of $\mathrm{O}\left(10^{-4}\right) \mathrm{m}^{2} / \mathrm{s}[14,15]$. The enormous amounts of mechanical energy (of order $2 \times 10^{12} \mathrm{~W}$ ) required to maintain such a large rate of vertical mixing are available only from the tides and the surface winds [15]. Consequently, it has been argued $[5,27,28]$ that the thermal buoyancy cannot be the dominant driving force for the overturning circulation and that heat stored in the relatively warm equatorial surface waters must be carried passively towards high latitudes by the circulation. However, the argument appears to confuse the forces providing momentum to the mean overturning flow with sources of energy for the vertical mixing.

Dissipation and tracer measurements in the ocean interior $[3,9$, $7,12]$ on the other hand support a much smaller vertical diffusivity (of order $10^{-5} \mathrm{~m}^{2} / \mathrm{s}$ ) than that required by existing models. Recent data [23] also suggest that the basin-averaged diffusivity is less than a factor of three above that in the interior, despite the discovery of regions of very intense turbulence associated with topography $[20,10,8]$. Here we show that, given the estimated meridional heat flux in the oceans, a simple and self-consistent energy argument predicts both a mechanical energy flux in agreement with general circulation models and inferences from data, and a vertical diffusivity in agreement with measurements in the ocean interior. We conclude that the observed circulation can be driven by buoyancy fluxes at the surface and need not be reliant on energy input to mixing from winds and tides. A dynamical model and solution for the circulation is also required to demonstrate that a flow satisfying the energy requirements is possible and consistent with observations, and this will be presented elsewhere.

\section{Available Potential Energy Fluxes due to Surface Heating}

In order to discuss the mechanical energy supplied to a box of fluid by differential heating along the surface (figure 1) we divide the flow driven into two regions - the surface boundary layer and the interior. Water in a portion of the surface boundary layer of depth $h$ is supplied with heat at a rate $q_{h}$ while heat is removed from the remainder of the surface waters at a rate $q_{c}\left(=-q_{h}\right)$. While we choose to describe the circulation in terms of the heat flux carried, this does not imply any particular boundary condition on heat flux or temperature. The hor- 
izontal variation of heat flux gives rise to a horizontal density gradient and flow (at a velocity $u$ ) within the surface layer. The consequent horizontal mass flux in the boundary layer must be ejected into the interior for heat throughput to be maintained in the flow. Mass is returned to the surface boundary layer by a broad upwelling from the interior, as shown in figure 1 . The boundary layer thickness $h$, which characterizes both the velocity and thermal structure, is therefore set by a balance between downward diffusion of heat from the surface and upward advection. Thus, conservation of heat in the boundary layer requires that

$$
q_{h}=-q_{c} \sim \rho_{r} c_{p} \kappa_{b} A_{h} \frac{\delta T}{h} \sim \rho_{r} c_{p} \frac{A}{L} \delta T u h,
$$

where $\delta T$ characterizes both the vertical and horizontal temperature variation in the surface boundary layer, $A$ and $L$ are the cross-sectional area and length of the box, respectively, $A_{h}$ is the cross-sectional area over which heating is applied, $\kappa_{b}$ is the thermal diffusivity in the boundary layer, $c_{p}$ is the specific heat, and $\rho_{r}$ is a reference density. If the vertical velocity is zero at the surface $(z=0)$ and $h \ll L$ (an assumption that will be justified in the next section), the equations of continuity and heat transport require that

$$
\frac{u}{L} \sim \frac{w}{h} \sim \frac{\kappa_{b}}{h^{2}} .
$$

Observations from laboratory experiments and numerical solutions for horizontal convection show that the ejection of mass flux from the surface boundary layer occurs in a localized plume $[21,22,18,13]$, the dynamics of which we will examine below. For the moment it is necessary only to realise that water in the plume must be negatively buoyant to be ejected into the interior. Diffusion in the interior, together with zero flux conditions on the sidewalls and lower boundary, ensures that the flow is in a steady state only if the plume penetrates to the bottom [13]. The temperature variation in the plume required to drive this flow is denoted as $\delta T^{\prime}$, which must also characterize the temperature variation in the interior. As water in the plume is relatively dense, the box has an amount of available potential energy (APE) equal to the potential energy that would be released by letting the fluid adjust adiabatically to static equilibrium. If $\alpha$ is the coefficient of thermal expansion and $g$ the gravitational acceleration, the buoyancy flux in the plume is $F^{\prime}=\rho_{r} g \alpha \delta T^{\prime} u h A / L$ and represents the rate at which excess weight appears in the plume at the base of the boundary layer. As the plume falls through a depth $H-h$ in reaching its level of neutral buoyancy, by equation (1) APE is generated at a rate

$$
\dot{P}^{\prime} \sim(H-h) F^{\prime}=\frac{\alpha g(H-h) q_{c}}{c_{p}} \frac{\delta T^{\prime}}{\delta T},
$$

where we have assumed the space occupied by the plume to be much less than the total volume of the box.

\section{Turbulent Mixing Energized by Convection}

We now consider the dissipation of energy, which for a steady state exactly balances the supply of mechanical energy (equation 3). It has been shown [18, equation 3.4] that in a box of Boussinesq fluid forced only by a temperature difference (or heat flux) imposed along its top boundary, the volume-averaged rate of viscous dissipation of energy per unit mass, $\bar{\varepsilon}$, is given by

$$
\bar{\varepsilon}=\kappa_{b} g \alpha \overline{\Delta T} / H,
$$

where the uptake of heat at the boundary is characterized by the diffusivity $\kappa_{b}$, (which was assumed to be the molecular diffusivity), and $\overline{\Delta T}$ is the temperature difference between horizontal averages at the top and bottom of the box. This result (equation 4) implies that the flow cannot be turbulent in the limit of

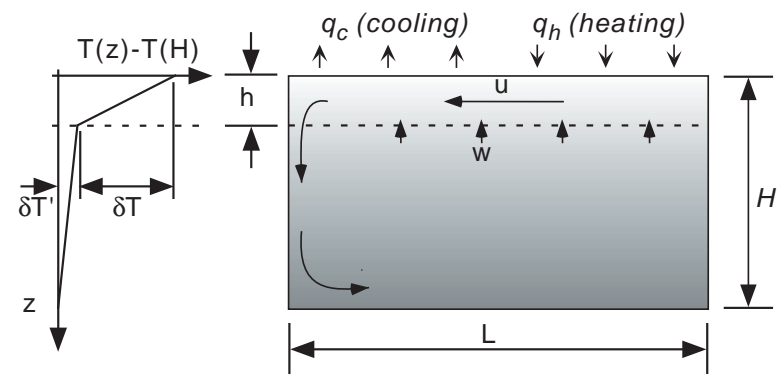

Figure 1: Schematic diagram of the convective flow driven in a box by differential heating along a horizontal boundary. Surface heat fluxes $q_{c}$ and $q_{h}\left(=-q_{c}\right)$ transfer heat to/from the boundary layer adjacent to the surface, maintaining a horizontal density gradient and a flow directed from the heated region to the cooled region. For sufficiently large heat fluxes most of the temperature variation occurs across the surface boundary layer, which occupies a small fraction of the total depth.

vanishing diffusivity or infinite fluid depth [18]. For densitystratified flow the vigour of turbulence can be measured by the parameter $\varepsilon / v N^{2}$, where $\varepsilon$ is the local rate of viscous dissipation per unit mass, $N=[-g \alpha(d T / d z)]^{1 / 2}$ is the local buoyancy frequency characterizing the density stratification and $z$ is the depth [1]. Hence from equation (4), for general values of the diffusivity, horizontal convection in water must have a volume averaged $\bar{\varepsilon} / v \overline{N^{2}} \approx \kappa_{b} / v$, which $\sim 0.1$ if $\kappa_{b}$ is set equal to the molecular diffusivity $\kappa$, no matter what the depth or heat flux. This value implies very weak or no turbulence [1].

On the other hand, equation (3) shows that for an imposed heat flux the total dissipation in the box (which must be equal to the mechanical energy input) increases in proportion to the heat flux $q_{c}$ and depth $H$, and is independent of the viscosity and molecular diffusivity. The average dissipation per unit mass is therefore independent of $H$ and inversely proportional to the area $A$. There is an apparent inconsistency with the results from equation (4). However, the value of $\bar{\varepsilon} / v \overline{N^{2}}$ obtained above from equation (4) is based on the overall depth-averaged buoyancy frequency. Hence, the constraints imposed by equations (3) and (4) may both be satisfied if for a given fluid (water) we propose that below the surface layer $(z>h)$ the density gradient becomes small and the flow becomes turbulent when $h \ll H$ (whereas for very large horizontal area, the temperature difference and dissipation rate vanish). Our later scaling analysis will indicate when this a priori assumption is invalid. We again characterize turbulent mixing in the density-stratified interior by a vertical diffusivity $\kappa^{*}$ and use the usual relationship $[17,3,7]$

$$
\kappa^{*}=\eta\left(\frac{\varepsilon}{N^{2}}\right)
$$

where $\eta$ can be interpreted as the ratio of the turbulent energy used for vertical mixing to the energy dissipated by viscosity. The ratio $\eta$ is commonly taken to be 0.2 [17], but an alternative formulation for weak turbulence [1] gives $\eta=0.47$ based on the molecular properties of seawater. The volume-averaged rate of viscous dissipation per unit mass will be given by

$$
\bar{\varepsilon} \approx \overline{\varepsilon^{\prime}}=\frac{1}{V^{\prime}} \int_{V^{\prime}} \varepsilon d V^{\prime}=\frac{\kappa_{i}^{*} g \alpha \delta T^{\prime}}{\eta(H-h)},
$$


where $V^{\prime}$ is the volume of the interior (excluding the surface layer) and $\bar{\varepsilon}^{\prime}$ is the volume-averaged rate of viscous dissipation per unit mass in the interior. We have assumed the crosssectional area $A$ to be independent of depth and defined an averaged interior diffusivity $\kappa_{i}^{*}$ (which applies below the boundary layer). Setting equations (4) and (6) to be equal gives

$$
\kappa_{i}^{*}=\kappa_{b} \eta\left(\frac{\overline{\Delta T}}{\delta T^{\prime}}\right)\left(\frac{H-h}{H}\right) .
$$

The total rates $\dot{W}_{p e}^{\prime}$ and $\dot{W}_{k e}^{\prime}$ at which work is done by turbulence in the interior against gravity and against viscosity, respectively, can be summed to give the total rate of working

$$
\begin{aligned}
\dot{W}^{\prime} & =\dot{W}_{k e}^{\prime}+\dot{W}_{p e}^{\prime} \\
& =\rho_{r} \int_{V^{\prime}} \varepsilon d V^{\prime}+g \int_{V^{\prime}} \kappa_{i}^{*} \frac{d \rho}{d z} d V^{\prime} \\
& =g A \kappa_{i}^{*} \frac{1+\eta}{\eta}[\bar{\rho}(H)-\bar{\rho}(h)],
\end{aligned}
$$

where $\rho$ is the density in the interior and the overbar denotes a horizontal average. Setting $\dot{W}^{\prime}$ in equation (8) equal to $\dot{P}^{\prime}$ in equation (3), along with $\rho=\rho_{0}\left[1-\alpha\left(T-T_{0}\right)\right]$, and then using equation (1) gives

$$
\begin{aligned}
\kappa_{i}^{*} & \sim\left(\frac{\eta}{1+\eta}\right)\left(\frac{(H-h) q_{c}}{\rho_{r} c_{p} A \delta T}\right) \\
& =\kappa_{b}\left(\frac{\eta}{1+\eta}\right)\left(\frac{H-h}{h}\right) \frac{A_{h}}{A},
\end{aligned}
$$

where $\delta T=\bar{T}(0)-\bar{T}(h)=\overline{\Delta T}-\delta T^{\prime}$. Combining equations (7) and (9) gives the temperature variation in the interior

$$
\frac{\delta T^{\prime}}{\overline{\Delta T}} \sim \frac{h}{H}(1+\eta) \frac{A}{A_{h}} .
$$

By equations (7), (9) and (10), the assumption of a turbulent interior (where $\kappa_{i}^{*}>\kappa, \kappa_{b} \geq \kappa$ ) is seen to be valid when the surface boundary layer depth is much smaller than the basin depth, i.e. $h / H \ll 1$. The temperature drop $\delta T$ across the surface boundary layer is then almost the same as the top-to-bottom temperature difference $\overline{\Delta T}$, where $\delta T$ and $h$ satisfy equation (1). As $H / L<1$ for the small aspect ratio basin geometry assumed here, the earlier assumption $h / L \ll 1$ will be automatically satisfied. The interior flow will become non-turbulent at sufficiently small heat fluxes, as given by equation (1) when the surface boundary layer occupies the full depth. The molecular viscous stresses dissipate energy per unit mass at a rate given by equation (4), which in the non-turbulent flow is also equal to the rate of energy input required to maintain the density field by molecular diffusion.

Applying the results to the experiments of Mullarney et al. [13], where the boundary layer at the forcing boundary was laminar we consider the case $q_{c}=140 \mathrm{~W}$, in which $\delta T \sim \overline{\Delta T} \approx 17^{\circ} \mathrm{C}$. Here equations (1), (9) and (10) predict $h \sim 0.007 \mathrm{~m}, \kappa_{i}^{*} \sim 0.4-$ $0.7 \times 10^{-6} \mathrm{~m}^{2} / \mathrm{s}$ and $\delta T^{\prime} \sim 1.4-1.7^{\circ} \mathrm{C}$ (for $\eta=0.2-0.47$ ). From equations (4) and (7), $\bar{\varepsilon} / v N_{i}^{2}=\kappa_{i}^{*} / v \eta \sim \mathrm{O}(3)$, which indicates very weak turbulence in the interior and a diffusivity 3 to 5 times the molecular value for heat $\left(A=2 A_{h}=0.1875\right.$ $\mathrm{m}^{2}, L=1.25 \mathrm{~m}, H=0.2 \mathrm{~m}, \kappa=1.47 \times 10^{-7} \mathrm{~m}^{2} / \mathrm{s}, \rho_{r}=1000$ $\left.\mathrm{kg} / \mathrm{m}^{3}, c_{p}=4184 \mathrm{~J} / \mathrm{kg} / \mathrm{K}, v=7.6 \times 10^{-7}\right) \mathrm{m}^{2} / \mathrm{s}$ ). These predictions compare reasonably well with measurements: $h=\mathrm{O}(0.01)$ $\mathrm{m}$ and $\delta T^{\prime}$ in the range $0.34-3.5^{\circ} \mathrm{C}$, noting that the actual edge of the boundary layer is not well-defined and that the temperature profiles were not taken in the plume itself.
In applying the results to the oceans, we first note that equation (1) would predict the surface boundary layer (i.e. thermocline) thickness to be only a few metres based on a molecular thermal diffusivity. Instead, we expect a turbulent thermocline whose properties are determined primarily by wind-driven processes. Thus if the effective diffusivity in the thermocline is $\kappa_{b}(\gg \kappa)$, the volume-averaged viscous dissipation per unit mass $\bar{\varepsilon}_{o}$ throughout the oceans is then a factor of $1 / \eta$ larger than when the buoyancy uptake at the surface is by molecular diffusion (equation 4 with $\kappa_{b}=\kappa$ ),

$$
\bar{\varepsilon}_{o}=\frac{\kappa_{b} g \alpha \overline{\Delta T}}{\eta H} .
$$

Viscous dissipation by turbulence in the thermocline must also be allowed for in the energy budget. Equating the total viscous dissipation rate to the sum of the viscous dissipation rates in the interior and thermocline,

$$
\int_{V} \bar{\varepsilon}_{o} d V=\int_{V^{\prime}} \varepsilon d V^{\prime}+\int_{V_{b}} \varepsilon d V_{b}
$$

and substituting equation (5) gives

$$
\kappa_{b} \overline{\Delta T}=\kappa_{i}^{*} \delta T^{\prime}+\kappa_{b} \delta T,
$$

where $V_{b}$ is the volume incorporating the thermocline. Noting that $\overline{\Delta T}=\delta T+\delta T^{\prime}$, equation (13) requires that the diffusivity is uniform throughout the flow, i.e. $\kappa_{b}=\kappa_{i}^{*}$. Physically this is because the diffusivity in the boundary layer is set by the external energy supplied (mainly from wind-driven turbulence). The total rate of working in the interior is still given by equation (8), but replacing $\kappa$ with $\kappa_{b}$ in equation (9) leads to the surprising conclusion that the thermocline thickness in this convection model is set by geometrical considerations alone, i.e.

$$
\frac{h}{H}=\frac{A_{h} / A}{A_{h} / A+(1+\eta) / \eta} .
$$

If heating is applied over one half of the surface area, as is approximately the case for the oceans $\left(A_{h} / A=0.5\right)$, equation (14) predicts $h / H \sim 0.08-0.14$ for $\eta=0.2-0.47$. Substituting equation (14) into equation (1) and taking $\alpha \delta T \approx \delta \rho / \rho_{r}$ gives

$$
\kappa_{i}^{*} \delta \rho=\frac{\alpha q_{c} H}{c_{p} A\left[A_{h} / A+(1+\eta) / \eta\right]},
$$

where $\delta \rho=\bar{\rho}(h)-\bar{\rho}(0)$. It can also be shown using equation (5) that the density ratio

$$
\frac{\delta \rho^{\prime}}{\delta \rho}=\frac{\bar{\varepsilon}^{\prime}(H-h)}{\bar{\varepsilon}_{b} h}
$$

where $\delta \rho^{\prime}=\bar{\rho}(h)-\bar{\rho}(H)$ and $\bar{\varepsilon}^{\prime}$ and $\bar{\varepsilon}_{b}$ are the volume-averaged rates of dissipation per unit mass in the interior (equation 4) and the thermocline, respectively.

As $\delta \rho^{\prime}+\delta \rho=\bar{\rho}(0)-\bar{\rho}(H)$, equation (16) would now close the problem if we knew the rate at which external energy was supplied to turbulence in the thermocline. However, without an estimate of this external input, we can only show that this scaling is consistent with measurements from the oceans. Taking $H=3780 \mathrm{~m}$, the average depth of the oceans [2], equation (14) predicts $h$ to be $300-530 \mathrm{~m}$. Using the globally averaged potential density profile from the Levitus 1994 dataset to estimate $\delta \rho^{\prime}$ and $\delta \rho$ for the predicted thermocline depth (http://iridl.ldeo.columbia.edu/SOURCES/.LEVITUS94/), we find $\delta \rho^{\prime} \sim 1.1-0.7 \mathrm{~kg} / \mathrm{m}^{3}, \delta \rho \sim 2.0-2.4 \mathrm{~kg} / \mathrm{m}^{3}$ (for $\eta=$ $0.2-0.47$ ). By equation (15), we then predict the diffusivity 
$\kappa_{i}^{*}=\kappa_{b}=2-3.2 \times 10^{-5} \mathrm{~m}^{2} / \mathrm{s}$. This agrees nicely with available measurements of vertical mixing [23]. We have taken the total heat loss $q_{c}$ from the oceans to be approximately $4 \times 10^{15}$ $\mathrm{W}([4, \mathrm{p} .212]$, and inferred from hydrographic data, [11]), the surface area of the oceans $A=3.6 \times 10^{14} \mathrm{~m}^{2}$ [2], $\rho_{r} \sim 1025$ $\mathrm{kg} / \mathrm{m}^{3}, c_{p} \approx 3990 \mathrm{~J} / \mathrm{kg} / \mathrm{K}$ for seawater at $20^{\circ} \mathrm{C}$ and atmospheric pressure [2] and $\alpha=2.54 \times 10^{-5} \mathrm{~K}^{-1}$ for seawater at $-2^{\circ} \mathrm{C}$ [2], a temperature representative of the Weddell Sea outflow source water. Furthermore we predict that turbulence in the oceans has an intensity $\bar{\varepsilon}^{\prime} / v N_{i}^{2}=\bar{\varepsilon} / v \overline{N^{2}}=\kappa_{i}^{*} / v \eta=\mathrm{O}(80)$. However, we have not yet considered the sinking regions, where there will be a larger-than-average dissipation rate.

\section{Energy Fluxes in a Simple Circulation Model and Applica- tion to the Oceans}

The above arguments are now applied to the ocean overturning by adopting an analytically more convenient model for the near-surface flow, a simple assumption about the interior flow, and recognising the deep sinking leg as a turbulent plume. Although the plume of interest in the oceans will be a slope current supplied by overflow from a sill, we discuss the simpler case of a vertical three-dimensional plume as shown in figure 2, where the plume originates at the surface. We first demonstrate that the energetics governing this situation are the same as those discussed for the more complex flow in figure 1. The structure of the surface layers in the oceans is more complicated than that sketched in figure 1. Heat transfer across the air-sea interface is not simply by molecular conduction, but also involves a combination of radiative and evaporative transfer, wave action and turbulence. We have demonstrated that the heat transfer in the water column is strongly influenced by external energy input (most probably through wind-driven turbulence). However, as we will show, forcing of the abyssal circulation is a result of the surface buoyancy flux.

We assume that the thermal forcing is constant on the overturning timescale. The rate of heat loss at the surface $q_{c}$ produces a total buoyancy flux

$$
F_{T}=\frac{\alpha g q_{c}}{c_{p}}
$$

that is the sum of buoyancy fluxes over all regions of deep downwelling, which we assume to be plumes (we later assume that these are turbulent, but this restriction is not required at this stage). The rate of generation of APE at any level is equal to the local buoyancy flux multiplied by the distance the plume still has to fall. Thus, for the plume in figure 2 that falls through a depth $D$,

$$
\dot{P}=D F=\frac{\alpha g D q_{c}}{c_{p}} .
$$

If this plume falls to the bottom, $D=H$. The flux of APE reduces rapidly through the surface layers to be equal to equation (3) at a depth $z=h$. Thus it is apparent that very little residual APE is required to drive the abyssal circulation. We proceed by applying the results for the abyssal circulation in figure 1 to the flow in figure 2 and we justify below why this approximation is valid.

Deep convection occurs in both the northern and southern hemispheres, but surface waters sink to the greatest depth at sites around the Antarctic to form Antarctic Bottom Water (AABW). Surface waters that sink in the north Atlantic are found in the abyssal ocean at levels above the AABW. This is consistent with the prediction, for turbulent plumes, that the plume with the greatest buoyancy flux reaches the bottom and dominates the density structure $[6,26]$. From equation $(18)$, this plume will also make the dominant contribution to the total $\dot{P}$. Therefore, we consider the flow due to a single southern hemisphere

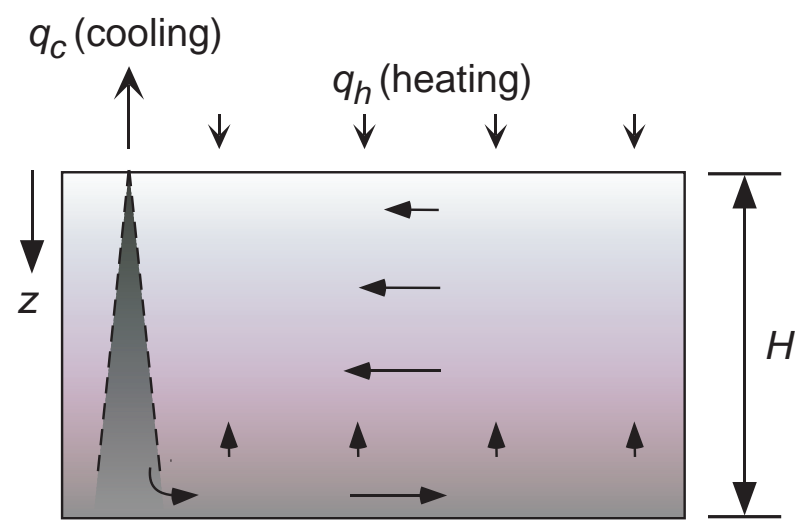

Figure 2: Schematic diagram of a model for horizontal convection (and the meridional overturning circulation of the oceans). Surface cooling at a rate $q_{c}$ leads to a small region of downwelling (represented as a vertical plume) from the surface to the bottom in an idealized basin of depth $H$ and cross-sectional area $A$. Heating at a rate $q_{h}=-q_{c}$ is distributed over the surface allowing the flow to reach a steady state. Only one downwelling (that with greatest buoyancy flux in the oceans) is shown. If the sinking is turbulent, entrainment drives recirculation at depth.

plume as a first approximation to the global ocean (figure 2). Equations (17) and (18) predict that the southern hemisphere heat loss $q_{c}$ of approximately $2 \times 10^{15} \mathrm{~W}[4,11]$, leads to a buoyancy flux of $F_{T} \approx 1.2 \times 10^{8} \mathrm{~N} / \mathrm{s}$ (or equivalently, after dividing by the density, $1.2 \times 10^{5} \mathrm{~m}^{4} / \mathrm{s}^{3}$ ), and APE generation at a rate of $\dot{P} \approx 0.47 \times 10^{12} \mathrm{~W}$. We have taken $D=H=3780 \mathrm{~m}$, $g=9.81 \mathrm{~m} / \mathrm{s}^{2}$ and properties for seawater as given earlier. The coefficient of expansion is strongly temperature dependent, but we have used that for seawater at $-2^{\circ} \mathrm{C}\left(\alpha=2.54 \times 10^{-5} \mathrm{~K}^{-1}\right.$ [2]) because the generation of available potential energy that may drive turbulence in the interior will be determined primarily by the density difference in the interior.

This predicted rate of APE generation compares with values calculated for the global ocean from an ocean general circulation model $\left(0.3 \times 10^{12} \mathrm{~W}[25]\right)$ and from observations of the density distribution and fluxes of heat and water at the surface $\left(1.4 \pm 0.8 \times 10^{12} \mathrm{~W}[16]\right)$. The latter value is based on small density gradients in the surface mixed layer and is likely to be an over-estimate [25]. The definition of the rate of generation (or flux) of potential energy (equation 18) avoids any estimates of the actual APE in the flow, and therefore does not require discussion of a reference energy state. Note, by equation (18), that the potential energy flux through the system is several thousand times smaller than the heat energy flux from which it arises, but that only the potential energy flux is of dynamical significance in the energy budget of the overturning circulation. In order to calculate the overturning circulation that could be supported by the input of available potential energy calculated above, let us assume that all of $\dot{P}$ is converted to kinetic energy and that turbulent mixing in the density-stratified ocean is characterized by a vertical diffusivity $\kappa^{*}$ that is the same at any point for heat, salt and, with a linear equation of state, also density [15]. For the situation in figure 2 , the total rate of working by turbulence against gravity and against viscosity evaluates to

$$
\begin{aligned}
\dot{W} & =\rho_{r} \int_{V} \varepsilon d V+g \int_{V} \kappa^{*} \frac{d \rho}{d z} d V \\
& =g A \overline{\kappa^{*}} \frac{1+\eta}{\eta}[\bar{\rho}(H)-\bar{\rho}(0)],
\end{aligned}
$$

where $V$ is the full volume (cf. equation 8). For a simple first 
approximation to the oceans, we have again assumed the crosssectional area $A$ to be independent of depth and defined an average diffusivity $\overline{\kappa^{*}}$ over the whole volume. Horizontal transports in the oceans are typically large enough to achieve horizontal density differences over most of the basin that are much smaller than the vertical difference [15], and we have implicitly assumed this to be the case. Recalling that $\overline{\Delta T}=\delta T+\delta T^{\prime}$, equations (3), (8), (18) and (19) give

$$
\frac{\dot{P}^{\prime}}{\dot{P}}=\frac{(H-h)}{H} \frac{\overline{\Delta T}}{\delta T} \frac{\overline{\kappa^{*}}}{\kappa_{i}^{*}} \frac{\dot{W}^{\prime}}{\dot{W}} \approx c \frac{\dot{W}^{\prime}}{\dot{W}} .
$$

For a laminar surface boundary layer with $h \ll H$, $\overline{\kappa^{*}}$ can be estimated by a volume average over the interior and boundary layer (i.e. $H \overline{\kappa^{*}}=h \kappa_{b}+(H-h) \kappa_{i}^{*}$ ) and equations (7), (9) and (10) give $c \approx 1$ in equation (21). For the turbulent surface boundary layer in the oceans, $c \approx 1.1(\eta=0.47)-1.4(\eta=0.2)$, using our earlier scaling results and $\overline{\kappa^{*}}=\kappa_{i}^{*}$. Thus equation (21) shows that although the rate of energy generation $\dot{P}$ in figure 2 is greater than the rate $\dot{P}^{\prime}$ for figure 1, the rate of dissipation $\dot{W}$ in figure 2 is correspondingly greater than $\dot{W}^{\prime}$ for figure 1 . Therefore, $\dot{P}-\dot{P}^{\prime} \approx \dot{W}-\dot{W}^{\prime}$, implying that the additional energy generated in the surface layer in figure 2 is also dissipated locally in the surface layer. Thus we expect the abyssal flows in figures 1 and 2 to be governed by equivalent energetics, and to not depend strongly on the detailed structure of the surface layers.

Equating $\dot{W}$ in equation (19) to $\dot{P}$ in equation (18) gives

$$
\overline{\kappa^{*}}=\left(\frac{\eta}{1+\eta}\right)\left(\frac{\alpha q_{c} H}{A c_{p} \overline{\Delta \rho}}\right)
$$

where $\overline{\Delta \rho}=\bar{\rho}(H)-\bar{\rho}(0)$. Using the above values for the southern hemisphere heat flux, the surface area and average depth of the oceans, the molecular properties of seawater, and the bottom-to-surface density difference from the globally averaged potential density $\left(\sigma_{0}\right)$ profile obtained from the Levitus 1994 dataset $\left(\bar{\rho}(H)-\bar{\rho}(0)=3.1 \mathrm{~kg} / \mathrm{m}^{3}\right)$, we again predict that turbulence could be supported in the ocean interior with an average diffusivity of $\overline{\kappa^{*}}=0.7 \times 10^{-5} \mathrm{~m}^{2} / \mathrm{s}(\eta=0.2)$ or $1.4 \times 10^{-5}$ $\mathrm{m}^{2} / \mathrm{s}(\eta=0.47)$. Although buoyancy is not expected to be the only energy source, these predictions are remarkably consistent with measurements of $\overline{\kappa^{*}}=\mathrm{O}\left(10^{-5}\right) \mathrm{m}^{2} / \mathrm{s}$ in the ocean interior $[3,9,7,12]$ and with the recent estimates of basin average diffusivities [23]. Of the available potential energy generated, $0.8-1.5 \times 10^{11} \mathrm{~W}$ is used to maintain the density structure.

\section{Conclusions}

Our theoretical analysis of the energetics of horizontal convection is consistent with our recent laboratory experiments [13], and is also consistent with the dissipation theorem of Paparella \& Young [18] in the case of a laminar surface boundary layer. The energy budget for the flow, along with a common-place relationship between turbulent mixing and viscous dissipation rates, leads to a prediction of the average interior mixing rate that is consistent with the measured ocean value. In this model, turbulent mixing in the interior is sustained as a consequence of the requirement that the upward diffusive buoyancy flux match the downward buoyancy flux in the sinking region. Thus the abyssal density gradient adjusts to allow sufficient vertical mixing. Among the factors that have not been taken into account is the seasonal salinity excess beneath sea ice, which will add to the buoyancy forcing at periods of maximum buoyancy flux. The tides and surface winds also will augment the buoyancydriven circulation calculated here by supplying energy to interior turbulence (at a rate that is poorly known), which increases the buoyancy uptake in the upper ocean. The spatial distribution of mixing from all three energy sources remains an open question.

\section{References}

[1] Barry, M. E., Ivey, G. N., Winters, K. B. and Imberger, J., Measurements of diapycnal diffusivities in stratified fluids, J. Fluid Mech., 442, 2001, 267-291.

[2] Gill, A. E., Atmosphere-Ocean Dynamics, Academic Press, London, England, 1982.

[3] Gregg, M. C., Scaling turbulent dissipation in the thermocline, J. Geophys. Res., 94, 1989, 9686-9698.

[4] Houghton, J. T., Meira Filho, L. G., Callander, B. A., Harris, N., Kattenberg, A. and Maskell, K., Climate change 1995: The science of climate change, Cambridge University Press, Cambridge, England, 1996.

[5] Huang, R. X., Mixing and energetics of the oceanic thermohaline circulation, J. Phys. Oceanogr., 29, 1999, 727746.

[6] Killworth, P. D. and Turner, J. S., Plumes with timevarying buoyancy in a confined region, Geophys. Astrophys. Fluid Dyn., 20, 1982, 265-291.

[7] Kunze, E. and Sanford, T. B., Abyssal mixing: where is it not?, J. Phys. Oceanogr., 26, 1996, 2286-2296.

[8] Ledwell, J. R., Montgomery, E. T., Polzin, K. L., Laurent, L. C. S., Schmitt, R. W. and Toole, J. M., Evidence for enhanced mixing over rough topography in the abyssal ocean, Nature, 403, 2000, 179-182.

[9] Ledwell, J. R., Watson, A. J. and Law, C. S., Evidence for slow mixing across the pycnocline from an open-ocean tracer release experiment, Nature, 364, 1993, 701-703.

[10] Lueck, R. G. and Mudge, T. D., Topographically induced mixing around a shallow seamount, Science, 276, 1997, 1831-1833.

[11] Macdonald, A. M., The global ocean circulation: a hydrographic estimate and regional analysis, Prog. Oceanogr., 41, 1998, 281-382.

[12] Matear, R. J. and Wong, C. S., Estimation of vertical mixing in the upper ocean at Station P from chlorofluorocarbons, J. Mar. Res., 55, 1997, 507-521.

[13] Mullarney, J. C., Griffiths, R. W. and Hughes, G. O., Convection driven by differential heating at a horizontal boundary, J. Fluid Mech., in press.

[14] Munk, W. H., Abyssal recipes, Deep-Sea Res., 13, 1966, 707-730.

[15] Munk, W. H. and Wunsch, C., Abyssal recipes II: energetics of tidal and wind mixing, Deep-Sea Res. I, 45, 1998, 1977-2010.

[16] Oort, A. H., Anderson, L. A. and Peixoto, J. P., Estimates of the energy cycle of the oceans, J. Geophys. Res., 99, 1994, 7665-7688.

[17] Osborn, T. R., Estimates of the local rate of vertical diffusion from dissipation measurements, J. Phys. Oceanogr., 10, 1980, 83-89. 
[18] Paparella, F. and Young, W. R., Horizontal convection is non-turbulent, J. Fluid Mech., 466, 2002, 205-214.

[19] Pierce, D. W. and Rhines, P. B., Convective building of a pycnocline: laboratory experiments, J. Phys. Oceanogr., 26, 1996, 176-190.

[20] Polzin, K. L., Toole, J. M., Ledwell, J. R. and Schmitt, R. W., Spatial variability of turbulent mixing in the abyssal ocean, Science, 276, 1997, 93-96.

[21] Rossby, H. T., On thermal convection driven by nonuniform heating from below: an experimental study, Deep-Sea Res., 12, 1965, 9-16.

[22] Rossby, H. T., Numerical experiments with a fluid nonuniformly heated from below, Tellus, 50, 1998, 242-257.

[23] Rudnick, D. L., Boyd, T. J., Brainard, R. E., Carter, G. S., Egbert, G. D., Gregg, M. C., Holloway, P. E., Klymak, J. M., Kunze, E., Lee, C. M., Levine, M. D., Luther, D. S., Martin, J. P., Merrifield, M. A., Moum, J. N., Nash, J. D., Pinkel, R., Rainville, L. and Sanford, T. B., From tides to mixing along the Hawaiian ridge, Science, 301, 2003, 355-357.

[24] Stommel, H., On the smallness of sinking regions in the ocean, Proc. Nat. Acad. Sci., Washington, 48, 1962, 766772 .

[25] Toggweiler, J. R. and Samuels, B., On the ocean's largescale circulation near the limit of no vertical mixing, $J$. Phys. Oceanogr., 28, 1998, 1832-1852.

[26] Wong, A. B. D. and Griffiths, R. W., Stratification and convection produced by multiple plumes, Dyn. Atmos. Oceans, 30, 2001, 101-123.

[27] Wunsch, C., Moon, tides \& climate, Nature, 405, 2000, 743-744.

[28] Wunsch, C. and Ferrari, R., Vertical mixing, energy, and the general circulation of the oceans, Ann. Rev. Fluid Mech., 36, 2004, 281-314. 